\title{
Valor en riesgo de crédito y déficit esperado aplicando cópulas
}

\author{
Credit Risk Value and Expected \\ Deficit Applying Copulas \\ Valor em risco de crédito e déficit \\ esperado aplicando cópulas \\ Alexander Andrade Cóndor \\ Universidad Andina Simón Bolívar, Sede Ecuador. Quito, Ecuador \\ alexander.andrade@uasb.edu.ec \\ https://orcid.org/0000-0003-1416-1123
}

DOI: https://doi.org/10.32719/25506641.2021.9.4

Recibido: 4 de marzo de 2020 - Revisado: 29 de junio de 2020

Aceptado: 11 de agosto de 2020

Artículo de investigación

Licencia Creative Commons

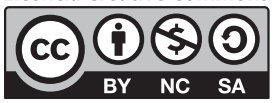




\section{Resumen}

Este trabajo presenta una aplicación de la teoría de cópulas a un portafolio de crédito de consumo ecuatoriano. Para la aplicación primero se estimaron las distribuciones marginales de la tasa de incumplimiento y del monto de exposición con base en la información histórica; luego, se construyeron cópulas y se aplicó el Teorema de Sklar a través de modelos de distribuciones multivariadas de cópulas (MVDC). Posteriormente, conociendo la estructura de dependencia, se estimó la pérdida total de la cartera, máxima pérdida, Credit VaR y Expected Shortfall (ES). Considerando un nivel de confianza de 99,5\%, en condiciones normales de mercado en un mes, la máxima pérdida que puede presentar el portafolio es de USD 18,65 millones (Credit VaR). De modificarse algún factor y empeorarse las condiciones de mercado, una vez superada la máxima pérdida, la pérdida esperada luego del Credit $\mathrm{VaR}$, es decir el ES, puede alcanzar un valor de USD 21,49 millones (15,22\% más que el Credit VaR). Finalmente, al comparar las estimaciones de los MVDC con la metodología del organismo de control ecuatoriano, se demostró que esta última subestima la pérdida esperada, indicadores de riesgo y eventos de pérdida extrema. Al no predecir los eventos extremos, se subestiman las pérdidas potenciales y aumenta el nivel del riesgo.

Palabras clave: cópula, riesgo de crédito, valor en riesgo de crédito, déficit esperado.

JEL: G24 Bancos de inversión, capital riesgo, corretaje.

\section{Abstract}

This paper presents an application of Copula Theory to an Ecuadorian consumer credit portfolio. To be applied, first, the marginal distributions of the default rate and the amount of exposure were estimated based on historical information; then copulas were built, and Sklar's Theorem was applied through Models of Multivariate Distribution of Copulas (MVDC). Subsequently, by knowing the dependency structure, the total loss of the portfolio, maximum loss, Credit VaR and Expected Shortfall (ES) were estimated. Considering a confidence level of $99,5 \%$ in normal market conditions in a month, the maximum loss that the portfolio can present is USD 18.65 million (Credit VaR). If any factor changes and market conditions worsen, once the maximum loss is exceeded, the expected loss after Credit VaR, that is, ES can reach a value of USD 21.49 million (15,22\% more than Credit VaR) . Finally, when comparing the estimates of the MVDC with the methodology of the Ecuadorian control body, it was shown that it underestimates the expected loss, risk indicators and extreme loss events. The failure to predict extreme events underestimates potential losses and increases risk levels.

Keywords: Copula, credit risk, value at credit risk, expected deficit.

JEL: G24 Investment banks, venture capital, brokerage. 


\section{Resumo}

Este trabalho apresenta uma aplicação da teoria de cópulas a um portfólio de crédito de consumo equatoriano. Para a aplicação, primeiramente se estimaram as distribuições marginais da taxa de inadimplência e do índice de exposição com base na informação histórica. Em seguida, construíram-se cópulas e aplicou-se o Teorema de Sklar por meio de Modelos de Distribuições Multivariadas de Cópulas (MVDC). Posteriormente, conhecendo a estrutura de dependência, estimou-se a perda total da carteira, a perda máxima, o Credit VaR e o Expected Shortfall (ES). Considerando um nível de confiança de 99,5\% em condições normais de mercado em um mês, a perda máxima que o portfólio pode apresentar é de USD 18,65 milhões (Credit VaR). Caso algum fator se modifique ou as condições de mercado piorem, uma vez superada a perda máxima, ou seja, a perda esperada após o Credit VaR, o ES pode alcançar um valor de USD 21,49 milhões (15,22 \% a mais que o Credit VaR). Finalmente, ao se compararem as estimativas dos MVDC com a metodologia do organismo de controle equatoriano, demonstrou-se que esta subestima a perda esperada, os indicadores de risco e os eventos de perda extrema. Ao não se preverem os eventos extremos, subestimam-se as perdas potenciais, aumentando o nível de risco.

Palavras-chave: Cópula, risco de crédito, valor em risco de crédito, déficit esperado. JEL: G24 Bancos de investimento, capital de risco, corretagem.

\section{Introducción}

L

a administración del portafolio de crédito reviste especial atención para los organismos de control debido al alto impacto macro y microeconómico, y a su capacidad de afectar la integralidad y regularidad de las instituciones financieras (Chang y Chi Chen 2010).

Las pérdidas ocasionadas por el riesgo de crédito, entendiéndolo como la pérdida potencial que se obtiene por motivos del incumplimiento en una transacción (Cerezo, Bielsa y Ramón 2011), son un tema central en el análisis de las instituciones financieras. No administrar correctamente este riesgo ocasiona pérdidas en el valor patrimonial de la compañía, problemas en la liquidez y solvencia.

Sheppard (2009) se refiere al riesgo de crédito como el evento en el cual una segunda parte no puede pagar los términos previamente acordados, por ello se lo conoce también como riesgo de incumplimiento. Choudhry (2006) indica cómo el incumplimiento de las obligaciones conlleva a las empresas a 
una pérdida igual a la cantidad adeudada por el deudor menos una cantidad de recuperación.

El riesgo de incumplimiento reviste mayor importancia en los portafolios de crédito en la medida en que la dispersión de las pérdidas dependen críticamente de la correlación entre los eventos de incumplimiento o default (Chang y Chi Chen 2010).

Por ello, en el Acuerdo de Basilea II (2006) se recomendó a los bancos y supervisores la estimación de requerimientos de capital de crédito a través de modelos internos basados en el sistema de administración de cada institución.

Al momento, el organismo de control del Ecuador estima la pérdida esperada multiplicando el nivel de exposición, severidad y probabilidad de incumplimiento ${ }^{1}$ (EC 2003, art. 2), sin considerar en el cálculo de la probabilidad la relación de los factores de riesgos, comportamiento histórico de los clientes, o modelos Credit Scoring. De ahí la necesidad de estudiar nuevas técnicas para mitigar las pérdidas en función al análisis de la estructura de dependencia del default.

En este sentido, además del análisis con modelos como el de Merton (1974), CreditMetrics (1977) o CreditPortafolio Manager de KMV, es necesario aplicar nuevos desarrollos teóricos que flexibilicen los supuestos de los modelos tradicionales, profundizando el análisis en escenarios dinámicos.

Bajo esta consideración, dentro de un portafolio existe una gran cantidad de factores de riesgo que interactúan constantemente entre sí. Así, para administrar un factor de riesgo se pueden identificar comportamientos marginales con técnicas univariadas. No obstante, dicho factor puede estar influido por el comportamiento de otros; de ahí que, los métodos que modelan la dependencia entre los factores de riesgo proporcionan valiosa información para la administración del portafolio.

En los modelos convencionales de asignación de portafolios como el CAMP, Capital Asset Princing Model, APT, entre otros, se utiliza como medida de dependencia el coeficiente de correlación de Pearson. Sin embargo, este presenta desventajas como el resumir en un solo número la relación de

1. La fórmula se encuentra en el Libro I, Título X.- De la gestión y administración de riesgos de la Superintendencia de Bancos del Ecuador (2004). 
dependencia, así como limitaciones cuando el grado de asociación no es lineal, es decir, es adecuado únicamente para el caso normal multivariado y es invariante solo ante transformaciones afines positivas (Embrechts, McNeil y Straumann 1999).

Por ello, para analizar los activos del portafolio se propone la función de cópula como una herramienta con una estructura flexible para caracterizar los diferentes tipos de dependencia (Melo y Becerra 2008). En términos de McNeil, Frey y Nyfeler (2001), las cópulas son funciones de distribución conjuntas de vectores aleatorios con distribuciones estándar marginales uniformes; su valor es proporcionar una forma de entender cómo las distribuciones marginales de los riesgos individuales se unen entre sí para formar distribuciones conjuntas (Embrechts, McNeil y Straumann 1999). Así, se propone el cálculo de la cópula como una medida que refleja la estructura de dependencia de los factores de riesgo (Boyé et al. 2000).

Las funciones de cópulas, además de permitir el estudio de la estructura de dependencia, brinda la facilidad de estimar indicadores como el valor en riesgo (VaR) y el déficit esperado (ES), con los cuales es posible mejorar la evaluación, administración y mitigación del riesgo de crédito.

El VaR es la máxima pérdida potencial en el valor de un portafolio con una probabilidad dada sobre un determinado período (Jadhav y Ramanathan 2009). Mientras que el ES es el promedio de las pérdidas superiores al VaR (Melo y Becerra 2008). El VaR responde a la pregunta qué tan malas pueden ser las pérdidas y ES responde a la pregunta cuánto es la pérdida esperada cuando las cosas van mal (Hull 2007). Por definición, la medida de riesgo ES es generalmente mayor que el VaR; esto conllevaría a un requerimiento de capital mayor que es consistente con lo esperado en el nuevo acuerdo de Basilea III (Mora 2010).

En este sentido, el objetivo de este documento es calcular y analizar la estructura de dependencia de un portafolio de crédito, mediante la aplicación de la teoría de cópulas para posteriormente estimar indicadores de riesgo para fortalecer la administración y mitigación del riesgo. 


\section{Revisión de la literatura}

Li (2000) inició con el estudio de la dependencia de los factores de riesgos analizando el problema de correlación del incumplimiento. Para estudiar la cartera de crédito especificó una distribución conjunta con distribuciones marginales conocidas a partir de la función de cópula. Posteriormente, Romano (2002) analizó la estructura de dependencia de una variable aleatoria con la función de cópula; luego, generó escenarios Montecarlo de los retornos de los factores de riesgo que afectan a una cartera de crédito. Sahamkhadam, Stephan y Östermark (2018) utilizaron las cópulas para analizar la estructura de dependencia en los rendimientos de mercados de valores.

Para disminuir el riesgo y mejorar la asignación de activos, Frey y McNeil (2002) desarrollaron el VaR para estimar la pérdida en la cartera de crédito. Clemente y Romano (2004) construyeron la distribución de pérdidas donde el momento hasta el incumplimiento de cada deudor se simula siguiendo un enfoque basado en cópulas. Con ello estimaron la pérdida esperada, máxima pérdida, el VaR y ES considerando su importancia como indicadores de riesgo coherentes, invariantes y legales (Chen 2018). Trabelsi y Tiwari (2019) demostraron que la función de cópula y el VaR son herramientas eficientes y atractivas para captar movimientos extremos.

Liu (2011), Choe y Jang (2011), Iscoe et al. (2012) y Li, Wang y Wang (2013) estimaron indicadores de riesgo analizando la estructura de dependencia para la medición del riesgo de crédito utilizando cópulas t de Student y Arquímedes señalando sus ventajas en comparación a las gaussianas.

Por su parte, Luo, Lu y Li (2015) demostraron que la cópula de Clayton es la más apropiada para describir alta dependencia en la cola, con ello estimaron un modelo VaR que mide con precisión el riesgo tanto en condiciones normales como en períodos de crisis; mientras que, Szetela, Mentel y Brożyna (2019) destacaron la ventaja de la cópula para modelar el incumplimiento con datos de alta dimensión y dependencia compleja.

En esta misma línea, Melo y Becerra (2008) analizaron un portafolio del mercado colombiano, evidenciando mejor desempeño de los resultados obtenidos con cópulas al considerar supuestos más reales y cuantificar de mejor manera el riesgo. 
En Ecuador, Maldonado y Pazmiño (2008) partiendo de los principales modelos de crédito utilizados en el ámbito internacional, ${ }^{2}$ propusieron un modelo general con base en funciones de cópulas. Con una cartera de crédito automotriz, determinaron que el modelo estimado con cópulas cuantifica de mejor manera las pérdidas extremas del portafolio.

Así también en México, Díaz y Ramírez (2009) estimaron el capital requerido para un portafolio de crédito considerando funciones de cópula y valores extremos, aquí se destaca la flexibilidad de la metodología, en especial de las cópulas elípticas generalizadas y agrupadas del tipo t de Student.

Finalmente, Díaz y Ramírez (2009) advirtieron sobre la efectividad del ajuste de cópulas en ausencia de datos confiables de largo plazo.

\section{Metodología}

El valor de la función de cópula es entender la estructura de dependencia sobre la base del comportamiento individual que se une para el análisis conjunto. En resumen, la metodología consiste en estimar distribuciones marginales, construir cópulas, aplicar el teorema de Sklar y estimar indicadores de riesgo.

A continuación, se presenta una descripción de las funciones y familias de cópula, considerando los trabajos de McNeil, Frey y Nyfeler (2001), y McNeil, Frey y Embrechts (2005).

Definición: una cópula d-dimensional es una función de distribución multivariada, $\mathrm{C}:[0,1]^{\mathrm{d}} \rightarrow[0,1]$ donde, sus distribuciones marginales (individuales) son uniformes estándar $\mathrm{U}_{1} \sim \mathrm{U}(0,1) \mathrm{i}=1,2 \ldots \mathrm{d}$.

Si una función $C$ satisface las siguientes propiedades, entonces $C$ es una cópula:

2. CreditMetricsTM, KMV, CreditRisk + Credit Porfolio View y Capitalización y Riesgo de Crédito (CyRCE). 
i) $C\left(u_{1}, \ldots, u_{d}\right)$ es crecientes en cada componente $u_{i}$.

ii) $C\left(1, \ldots, 1, u_{i}, 1, \ldots, 1\right)=u_{i}$ para todo $i \in\{1,2, \ldots, d\}, u_{i} \in[0,1]$.

iii) Para todo $\left(a_{1}, \ldots, a_{d}\right),\left(b_{1}, \ldots, b_{d}\right) \in[0,1]^{d}$ con $a_{i} \leq b_{i}$ se tiene que:

$$
\begin{aligned}
& \sum_{i 1=1}^{2} \cdots \sum_{i d=1}^{2}(-1)^{i_{1}+\cdots+i_{d}} C\left(u_{1 i_{1}}, \ldots, u_{d i_{d}}\right) \geq 0 \\
& \text { donde } u_{j 1}=a_{j} \text { y } u_{j 2}=b_{j} \text { para } j \in\{1,2, \ldots, d\} .
\end{aligned}
$$

Las cópulas elípticas están asociadas a variables aleatorias cuya función de distribución multivariada es de la forma $\mathrm{f}(\mathrm{x}$ 'x). Así, las curvas de nivel de variables aleatorias con este tipo de cópulas forman elipses (Boyé et al. 2000). En esta familia las cópulas más importantes son la Gaussiana y $t$ de Student (Cherubini, Luciano y Vecchiato 2004).

También existen cópulas de Arquímedes, que se pueden representar así:

$$
C\left(u_{1}, u_{2}\right)=\phi^{[-1]}\left(\phi\left(u_{1}\right)+\phi\left(u_{2}\right)\right)
$$

donde: $\phi$ se conoce como el "generador" de la cópula $C$ y $\phi^{[-1]}$ es la pseudoinversa de $\phi$ definida como:

$$
\phi^{[-1]}(s)=\left\{\begin{array}{c}
\phi^{-1}(s), \text { si } 0 \leq s \leq \phi(0) \\
0, \text { si } \phi(0) \leq s \leq+\infty
\end{array}\right.
$$

Las cópulas de Arquímedes más relevantes son:

- Cópula de Clayton, con el generador $\phi(t)=\frac{1}{\phi}\left(t^{-0}-1\right) \operatorname{con} \theta>0$. Formalmente:

$$
C^{G u}\left(u_{1}, \ldots, u_{d}\right)=\exp \left(-\left(\tilde{u}_{1}^{\theta}+\cdots+\tilde{u}_{d}^{\theta}\right)^{\frac{1}{\theta}}\right)
$$

- Cópula de Gumbel, su generador se define por $\phi(t)=\left(-\log (t)^{\theta} \operatorname{con} \theta>1\right.$. Formalmente:

$$
C^{C L}\left(u_{1}, \ldots, u_{d}\right)=\left(u_{1}^{-\theta}+\cdots+u_{d}^{-\theta}-d+1\right)^{\frac{1}{\theta}}
$$

donde: $\tilde{u}_{1} \equiv-\log u_{i}$.

Sklar (1959) reveló que se puede comprender la estructura de dependencia de un vector aleatorio mediante la cópula, por cuanto toda función de distribución multivariada de variables aleatorias continuas posee una cópula 
asociada, y la función de distribución multivariada puede ser construida mediante la cópula y sus funciones de distribución marginales.

A continuación, se presenta la definición formal del teorema:

Teorema de Sklar, sea $\mathrm{F}\left(\mathrm{r}_{1}, \ldots \mathrm{r}_{\mathrm{d}}\right)$ la función de distribución conjunta de las variables aleatorias $\mathrm{R}_{1} \ldots \mathrm{R}_{\mathrm{d}}$ con funciones de distribución marginales $\mathrm{F}_{1}$ $\left(\mathrm{r}_{1}\right) \ldots \mathrm{F}_{\mathrm{d}}\left(\mathrm{r}_{\mathrm{d}}\right)$. Existe una función $\mathrm{C}:[0,1] \mathrm{d} \rightarrow[0,1]$ tal que para todo $\mathrm{r}_{1} \ldots \mathrm{r}_{\mathrm{d}}$ :

$$
F\left(r_{1}, \ldots, r_{d}\right)=C\left(F_{1}\left(r_{1}\right), \ldots, F_{d}\left(r_{d}\right)\right)
$$

Si las funciones marginales son continuas, entonces $C$ es única; de otra forma, $C$ se encuentra definida únicamente en el $\operatorname{Ran}\left(F_{1}\right) \times \ldots \times \operatorname{Ran}\left(F_{\mathrm{d}}\right)$, donde $\operatorname{Ran}\left(F_{1}\right)$ se refiere al rango de la función $F_{1}$. De la misma manera, si $C$ es una cópula y $F_{1}\left(\mathrm{r}_{1}\right) \ldots F_{\mathrm{d}}\left(\mathrm{r}_{\mathrm{d}}\right)$ son funciones de distribución univariadas, entonces $F\left(\mathrm{r}_{1} \ldots \mathrm{r}_{\mathrm{d}}\right)$ definida en (1.1) es una función de distribución conjunta con distribuciones marginales $F_{1}\left(\mathrm{r}_{1}\right) \ldots F_{\mathrm{d}}\left(\mathrm{r}_{\mathrm{d}}\right)$.

Una vez identificada la estructura de dependencia, para estimar indicadores de riesgo se definió el incumplimiento o default $Y_{i}$. Suponiendo un portafolio que contiene $n$ activos, el incumplimiento para cada uno de los créditos $i$ se define como (Romano 2002):

$$
Y_{i}=\left\{\begin{array}{c}
1 \text { si para el crédito } i d>d_{d} \\
0 \text { caso contrario }
\end{array}\right.
$$

Donde, $\mathrm{d}=$ días de mora del crédito $i ; \mathrm{d}_{\mathrm{d}}=$ número de días de mora máximo que puede tener un crédito para no ser considerado como incumplido.

Luego, considerando que la pérdida esperada del portafolio se encuentra condicionada a la distribución multivariada del incumplimiento, se ajustó la tasa de incumplimiento con información histórica (Díaz y Ramírez 2009):

$$
T I_{t}=\frac{m_{t}}{M_{t}}
$$

Donde, $\mathrm{TI}_{t}=$ tasa de incumplimiento en el tiempo $t ; \mathrm{m}_{\mathrm{t}}=$ número de créditos incumplidos en el momento $t$.

$$
m_{t}=\sum_{i=1}^{n} Y_{i t}
$$

$\mathrm{mt}=$ número total de créditos del portafolio en el momento $t$. 
Como no es posible realizar un análisis de dependencia entre créditos incumplidos individualmente, por cuanto no se originan en el mismo momento, calcular la matriz de correlación necesaria para determinar el grado de dependencia resulta complicado para una gran cantidad de créditos; surge entonces la necesidad de construir grupos homogéneos dentro del portafolio.

Por ello, para la identificación y homogenización de los grupos del portafolio, se utilizó la técnica de Detección de Interacción Automática $J i$ - Cuadrada (CHAID), la cual mediante árboles de decisión segmenta e identifica divisiones óptimas cuando las variables son continuas, nominales o categóricas. El CHAID se utiliza comúnmente para el análisis del riesgo por su notable flexibilidad para identificar divisiones óptimas y la posibilidad de definir una categoría objetivo (Bickel et al. 1991).

Por medio de la siguiente expresión, se determinaron grupos homogéneos y se estimó la pérdida del portafolio (Díaz y Ramírez 2009):

$$
L_{g j}=T I_{g j} * E A D_{g j} * L G D
$$

Donde: $g=1,2,3 \ldots, G$ grupos; $\mathrm{L}_{\mathrm{gj}}=$ pérdida esperada del grupo $g$ en el escenario $j ; T I_{g j}=$ tasa de incumplimiento del grupo $g$ en el escenario $j$; EA$\mathrm{D}_{\mathrm{g}}=$ monto de exposición del grupo $g$ en el escenario $j ; \mathrm{LGD}=$ pérdida por el incumplimiento de los créditos que se supone no es estocástica. Cuantifica la porción de pérdida que el portafolio sufre en el caso de incumplimiento.

En el caso simple, se tienen escenarios $s$ en los cuales las contrapartes pueden incumplir o no con sus obligaciones. Para cada uno de los escenarios, la pérdida se definió por Clemente y Romano (2004):

$$
L_{j}=\sum_{g=1}^{G} L_{g j}
$$

Donde: $g=1 \ldots, G, j=1 \ldots s$ y $\omega_{i}=\left[\omega_{1} \ldots, \omega_{\mathrm{n}}\right]$ ' es el vector de posiciones del portafolio. En el momento $T$, la pérdida esperada total del portafolio para el escenario $j$ fue:

$$
E L(\omega)=\frac{1}{s} \sum_{j=1}^{s} L_{j}(\omega)
$$


EL $(\omega)$ corresponde a la pérdida promedio que se puede presentar en el portafolio.

Así también, se estimó el valor en riesgo (VaR) de crédito o CreditVaR, mediante la siguiente expresión (Clemente y Romano 2004):

$$
\text { CreditVaR }_{\alpha}=M L_{\alpha}(\omega)-E L(\omega)
$$

Donde: $\alpha=$ un nivel de confianza dado y $\operatorname{ML}_{\alpha}(\omega)=$ el $\alpha$-ésimo cuantil de la distribución de $\mathrm{L}_{j}$. $\mathrm{ML}_{\alpha}(\omega)=$ la máxima pérdida del portafolio a un nivel de probabilidad $\alpha$.

Como medida de riesgo extremo se calculó el déficit esperado o Expected Shortfall (ES). El ES es la pérdida esperada luego de superar las pérdidas mayores a la máxima pérdida del portafolio a un nivel de probabilidad $\alpha$ (Clemente y Romano 2004):

$$
E S_{s}(\omega)=M L_{\alpha}(\omega)+\frac{1}{(1-\alpha) s} \sum_{j=1}^{s}\left[L_{j}(\omega)-M L_{\alpha}(\omega)\right] I_{\left\{L_{j}(\omega)-M L_{\alpha}(\omega)>0\right\}}
$$

Siguiendo a Li (2000), la distribución de pérdidas del portafolio se obtuvo con técnicas de Montecarlo simulando posibles eventos crediticios como el incumplimiento de una obligación.

\section{Resultados}

Para aplicar la metodología se dispuso de un portafolio de consumo de una institución financiera ecuatoriana con información de cinco años. A la fecha de corte, el portafolio tenía 6267 créditos, la tasa de incumplimiento (TI) promedio fue 9,9\% con un monto de exposición de USD 23,4 millones.

Se definió como default a los créditos con más de 15 días de mora ${ }^{3}$ en al menos un mes:

3. Libro I.- Título IX.- De los activos y de los límites de crédito, bancos y seguros de la Superintencia de Bancos del Ecuador (2011). 


\section{$Y_{i}=\left\{\begin{array}{c}1 \text { si para el crédito } i d>d_{d} \\ 0 \text { caso contrario }\end{array}\right.$}

Donde: $d=$ días de mora del crédito $i ; \mathrm{d}_{\mathrm{d}}=15$ días.

Tomando como variable dependiente el incumplimiento $\mathrm{Y}_{i} \mathrm{y}_{\mathrm{y}}$ como variables independientes, el monto, plazo, tasa de interés y estado civil, utilizando la razón de verosimilitud y como categoría objetivo el default $\mathrm{Y}_{i}=1$, el CHAID identificó ocho grupos homogéneos como se demuestra en los nodos terminales del gráfico 1 .

La segmentación CHAID posee una estimación de riesgo del 0,112, lo cual indica que el modelo clasifica de forma correcta al 88,8 \% de las operaciones.

Para obtener las distribuciones marginales a partir de las distribuciones univariadas de la tasa de incumplimiento (TI) y monto de exposición (EAD) por grupo, se utilizó información histórica. Se ajustaron distribuciones con el método de máxima verosimilitud; posteriormente, se estimó la bondad de ajuste por medio de los estadísticos Kolmogorov-Smirnov (KS), Cramer-von Mises (CV), Anderson-Darling (AD); y complementariamente, se calcularon los criterios de información de Aikake (AIC) y Bayesiano (BIC). Para seleccionar la mejor distribución se consideraron los valores más bajos de los estadísticos de ajuste y criterios de información.

Partiendo de las distribuciones de probabilidad que la International Cost Estimating and Analysis Association sugiere para el análisis de riesgo (Braxton y Manning 2011), se consideraron catorce distribuciones. ${ }^{4}$ La distribución triangular se ajustó mejor demostrando así la flexibilidad de su forma, junto con la naturaleza intuitiva de la definición de sus parámetros y rapidez de uso (Fiorito 2006).

Para la construcción y estimación de los parámetros de las cópulas se utilizó el método de los momentos y sus correlaciones de rango. Debido a que el propósito es modelar el incumplimiento y estimar las pérdidas, considerando la posibilidad de eventos extremos, se estimaron varias clases de cópulas (Boyé et al. 2000).

4. Cauchy, Ji cuadrado, Exponencial, F. Gamma, Gumbel, Log Normal, Logística, Lognormal, Normal, t de Student, Triangular, Uniforme y Weibull. 


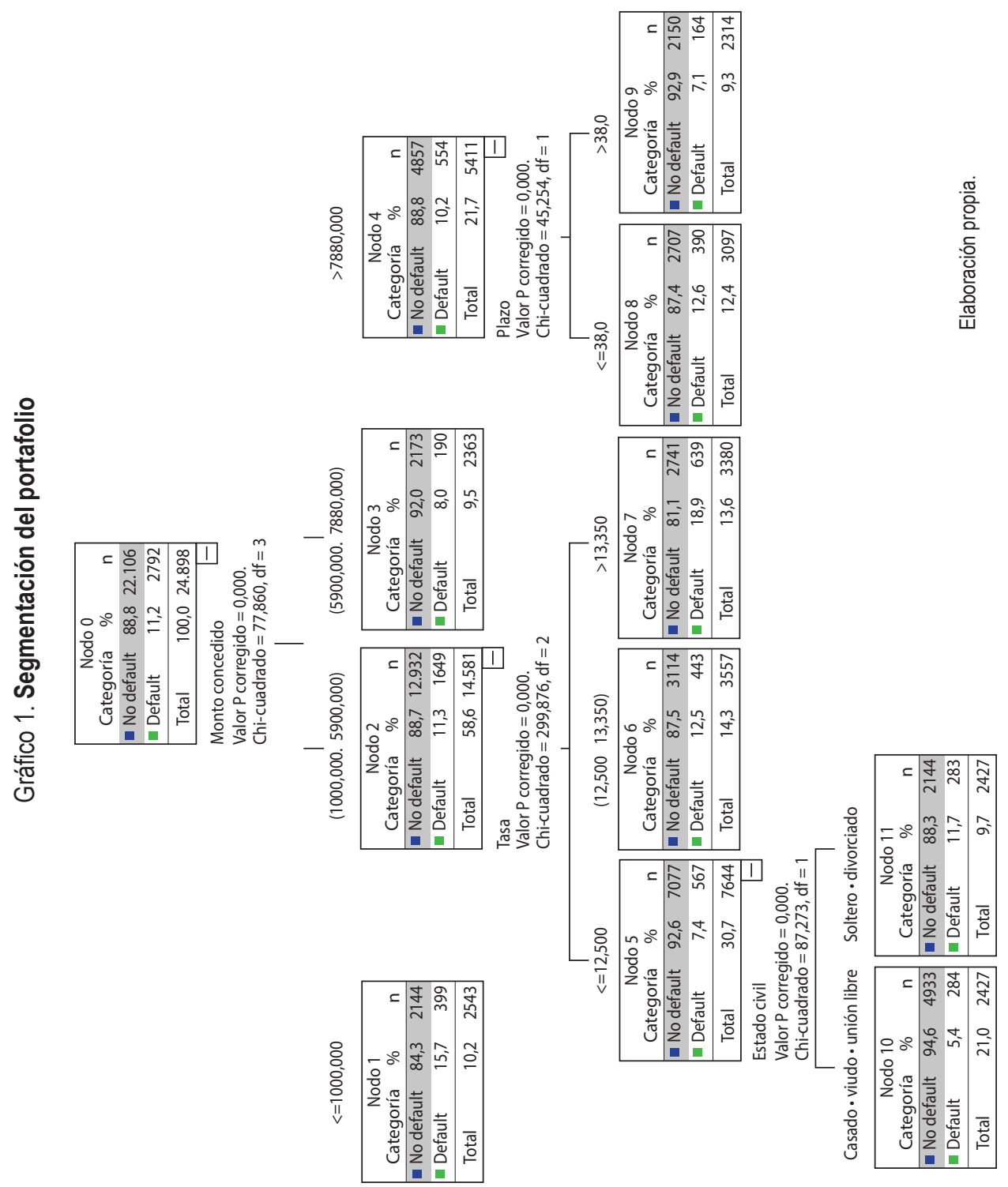


El primer paso para la construcción de la cópula fue determinar la correlación de rango entre la TI y el EAD mediante el método de Spearman. Posteriormente, se procedió a estimar el parámetro Rho por clase de cópula, para luego estimar las cópulas Normal, t de Student, de Frank, de Clayton y Gumbel para cada grupo.

Los resultados reflejan una relación positiva entre la TI y el EAD en los grupos uno, tres y cuatro, y una correlación negativa en el resto de los grupos. La correlación negativa evidencia resultados alentadores en cuanto a la administración de riesgo, por cuanto, mientras más alta sea la TI, menor será el EAD.

Para conocer el grado de ajuste, se realizaron 1000 simulaciones con cada clase de cópula y se obtuvo el Log de máxima verosimilitud (LMV) y criterios de información (AIC, BIC y Hannan y Quinn - HQ-).

Observando el valor más alto de LMV y los valores más bajos de los criterios AIC, BIC y HQ, el mejor ajuste se obtuvo con la cópula de Clayton, ${ }^{5}$ con excepción del grupo cinco, donde se seleccionó la cópula de Gumbel. Aquí, es necesario señalar que la cópula Clayton asigna mayor probabilidad a los eventos ocurridos en la cola izquierda, lo cual permite describir adecuadamente la dependencia negativa (Plascencia 2012); así también, genera distribuciones con mayor dependencia en las colas (Mensah 2020).

5. Con respecto al grupo tres, se seleccionó la cópula de Clayton por cuanto fue la única que presentó valores significativos en la prueba de dependencia multivariada, la cual se presenta más adelante. 
Tabla 1

Indicadores de ajuste de cópulas

\begin{tabular}{|c|c|c|c|c|c|}
\hline Grupo & Cópula & Log-Likelihood & AIC- & BIC- & HQ \\
\hline \multirow{5}{*}{ G1 } & Normal & 745,2224 & $-1488,4449$ & $-1483,5371$ & $-1476,6293$ \\
\hline & $\mathrm{t}$ de Student & 788,5106 & $-1573,0212$ & $-1563,2057$ & $-1549,3901$ \\
\hline & Clayton & 912,8611 & $-1823,7223$ & $-1818,8145$ & $-1811,9067$ \\
\hline & Frank & 664,1597 & $-1326,3194$ & $-1321,4116$ & $-1314,5039$ \\
\hline & Gumbel & 816,7800 & $-1631,5599$ & $-1626,6522$ & $-1619,7444$ \\
\hline \multirow{5}{*}{ G2 } & Normal & 884,4873 & $-1766,9746$ & $-1762,0669$ & $-1755,1591$ \\
\hline & $\mathrm{t}$ de Student & 892,1763 & $-1780,3526$ & $-.770,5371$ & $-1756,7216$ \\
\hline & Clayton & 1039,1782 & $-2076,3564$ & $-2071,4486$ & $-2064,5409$ \\
\hline & Frank & 760,8082 & $-1519,6163$ & $-1514,7086$ & $-1507,8008$ \\
\hline & Gumbel & 977,4568 & $-1952,9135$ & $-1948,0058$ & $-1941,0980$ \\
\hline \multirow{5}{*}{ G3 } & Normal & 5,5249 & $-9,0498$ & $-4,1420$ & 2,7657 \\
\hline & t de Student & 41,0612 & $-78,1223$ & $-68,3068$ & $-54,4913$ \\
\hline & Clayton & 8,8515 & $-15,7029$ & $-10,7952$ & $-3,8874$ \\
\hline & Frank & 2,7517 & $-3,5035$ & 1,4043 & 8,3120 \\
\hline & Gumbel & 12,2256 & $-22,4513$ & $-17,5435$ & $-10,6358$ \\
\hline \multirow{5}{*}{ G4 } & Normal & 260,3530 & $-518,7060$ & $-513,7983$ & $-506,8905$ \\
\hline & t de Student & 292,7394 & $-581,4789$ & $-571,6633$ & $-557,8478$ \\
\hline & Clayton & 330,9584 & $-659,9169$ & $-655,0091$ & $-648,1014$ \\
\hline & Frank & 224,8814 & $-447,7628$ & $-442,8550$ & $-435,9473$ \\
\hline & Gumbel & 329,6128 & $-657,2255$ & $-652,3177$ & $-645,4100$ \\
\hline \multirow{5}{*}{ G5 } & Normal & 86,7562 & $-171,5124$ & $-166,6047$ & $-159,6969$ \\
\hline & $\mathrm{t}$ de Student & 116,2058 & $-228,4117$ & $-218,5962$ & $-204,7807$ \\
\hline & Clayton & 111,1678 & $-220,3357$ & $-215,4279$ & $-208,5201$ \\
\hline & Frank & 65,1759 & $-128,3518$ & $-123,4440$ & $-116,5363$ \\
\hline & Gumbel & 116,4544 & $-230,9088$ & $-226,0010$ & $-219,0932$ \\
\hline
\end{tabular}




\begin{tabular}{|c|l|r|r|r|r|}
\hline Grupo & \multicolumn{1}{|c|}{ Cópula } & Log-Likelihood & \multicolumn{1}{c|}{ AIC- } & \multicolumn{1}{c|}{ BIC- } & HQ \\
\hline \multirow{4}{*}{ G6 } & Normal & 1591,3857 & $-3180,7715$ & $-3175,8637$ & $-3168,9559$ \\
\cline { 2 - 6 } & t de Student & 1595,4448 & $-3186,8895$ & $-3177,0740$ & $-3163,2585$ \\
\cline { 2 - 6 } & Clayton & 1780,1692 & $-3558,3384$ & $-3553,4306$ & $-3546,5229$ \\
\cline { 2 - 6 } & Frank & 1407,3823 & $-2812,7647$ & $-2807,8569$ & $-2800,9492$ \\
\cline { 2 - 6 } & Gumbel & 1692,2452 & $-3382,4905$ & $-3377,5827$ & $-3370,6750$ \\
\hline \multirow{4}{*}{ G7 } & Normal & 654,9713 & $-1307,9427$ & $-1303,0349$ & $-1296,1272$ \\
\cline { 2 - 6 } & t de Student & 665,7588 & $-1327,5175$ & $-1317,7020$ & $-1303,8865$ \\
\cline { 2 - 6 } & Clayton & 782,7438 & $-1563,4876$ & $-1558,5798$ & $-1551,6721$ \\
\cline { 2 - 6 } & Frank & 559,9110 & $-1117,8220$ & $-1112,9143$ & $-1106,0065$ \\
\cline { 2 - 6 } & Gumbel & 741,4125 & $-1480,8249$ & $-1475,9172$ & $-1469,0094$ \\
\hline \multirow{5}{*}{ G8 } & Normal & 497,9012 & $-993,8024$ & $-988,8947$ & $-981,9869$ \\
\cline { 2 - 6 } & t de Student & 511,9639 & $-1019,9278$ & $-1010,1123$ & $-996,2968$ \\
\cline { 2 - 6 } & Clayton & 599,1116 & $-1196,2232$ & $-1191,3154$ & $-1184,4076$ \\
\cline { 2 - 6 } & Frank & 423,5108 & $-845,0216$ & $-840,1138$ & $-833,2061$ \\
\cline { 2 - 6 } & Gumbel & 574,4625 & $-1146,9250$ & $-1142,0173$ & $-1135,1095$ \\
\hline
\end{tabular}

Elaboración propia.

Para demostrar el cumplimiento de las propiedades teóricas de las cópulas, es necesario verificar la uniformidad de las distribuciones marginales mediante la prueba de Anderson Darling, cuya Ho: $X \sim$ Uniforme $(0,1)$. En la tabla 2 se observa que en todos los casos las distribuciones generadas son uniformes.

Adicionalmente, se estimó una prueba de bondad de ajuste comparando la cópula empírica con una estimación paramétrica de la cópula derivada bajo una determinada hipótesis nula. Para obtener los p-valores aproximados se utiliza un bootstrap paramétrico (Genest, Rémillard y Beaudoin 2009). La hipótesis nula $H o$ para los grupos uno, dos, tres, cuatro, seis y siete fue la cópula de Clayton y para el grupo cinco fue la cópula de Gumbel. En la tabla 3 se observa que no se rechaza la $H o$, es decir, las cópulas seleccionadas son las correctas. 
Tabla 2

Prueba de Anderson Darling

\begin{tabular}{|c|c|c|c|c|}
\hline Grupo & Cópula & $\begin{array}{c}\text { Distribución } \\
\text { marginal }\end{array}$ & Anderson-Darling $\mathrm{t}$ & $p$-valol \\
\hline \multirow{2}{*}{ G1 } & \multirow{2}{*}{ Clayton } & $\mathrm{TI}$ & 0,6437 & 0,6076 \\
\hline & & EAD & 0,3924 & 0,8562 \\
\hline \multirow{2}{*}{ G2 } & \multirow{2}{*}{ Clayton } & $\mathrm{TI}$ & 0,209 & 0,9877 \\
\hline & & EAD & 0,9642 & 0,3766 \\
\hline \multirow{2}{*}{ G3 } & \multirow{2}{*}{ Clayton } & $\mathrm{TI}$ & 0,209 & 0,9877 \\
\hline & & EAD & 1,1818 & 0,2746 \\
\hline \multirow{2}{*}{ G4 } & \multirow{2}{*}{ Clayton } & $\mathrm{TI}$ & 0,209 & 0,9877 \\
\hline & & EAD & 0,653 & 0,5993 \\
\hline \multirow{2}{*}{ G5 } & \multirow{2}{*}{ Gumbel } & $\mathrm{TI}$ & 1,1399 & 0,2915 \\
\hline & & EAD & 0,6948 & 0,5632 \\
\hline \multirow{2}{*}{ G6 } & \multirow{2}{*}{ Clayton } & $\mathrm{TI}$ & 0,209 & 0,9877 \\
\hline & & EAD & 0,653 & 0,5993 \\
\hline \multirow{2}{*}{ G7 } & \multirow{2}{*}{ Clayton } & $\mathrm{TI}$ & 0,209 & 0,9877 \\
\hline & & EAD & 0,757 & 0,5131 \\
\hline \multirow{2}{*}{ G8 } & \multirow{2}{*}{ Clayton } & $\mathrm{TI}$ & 0,209 & 0,9877 \\
\hline & & EAD & 0,5207 & 0,7258 \\
\hline
\end{tabular}

Elaboración propia.

Tabla 3

Prueba bondad de ajuste

\begin{tabular}{|c|c|c|c|}
\hline Grupo & Cópula & Cramer-von Mises statistic & p-valor \\
\hline G1 & Clayton & 0,0197 & 0,2672 \\
\hline G2 & Clayton & 0,0209 & 0,2063 \\
\hline G3 & Clayton & 0,0136 & 0,8676 \\
\hline G4 & Clayton & 0,0162 & 0,6269 \\
\hline G5 & Gumbel & 0,0248 & 0,2073 \\
\hline G6 & Clayton & 0,0099 & 0,5440 \\
\hline G7 & Clayton & 0,0189 & 0,3382 \\
\hline G8 & Clayton & 0,0191 & 0,2409 \\
\hline
\end{tabular}

Elaboración propia. 
Una vez determinadas las distribuciones univariadas de TI y EAD, conociendo las distribuciones marginales y los parámetros de las cópulas, se aplicó el teorema de Sklar o MVDC, es decir, se construyó las distribuciones multivariadas con la estructura de dependencia de las cópulas considerando $\mathrm{n}=150$ escenarios.

Como ejemplo se muestran los resultados del Grupo 1, cuyo MVDC se construyó con una cópula de Clayton de parámetro 10,5648, y distribuciones marginales Triangular $(0,0018 ; 0,0497 ; 0,1791)$ para TI y Triangular $(78,2730 ; 132,661 ; 398,7994)$ para EAD. En los gráficos 2,3 y 4 se presentan los escenarios obtenidos con la cópula, sus distribuciones marginales, así como los gráficos de contorno y perspectiva de la superficie del MVDC.

\section{Gráfico 2}

\section{Escenarios y densidad de las distribuciones marginales}

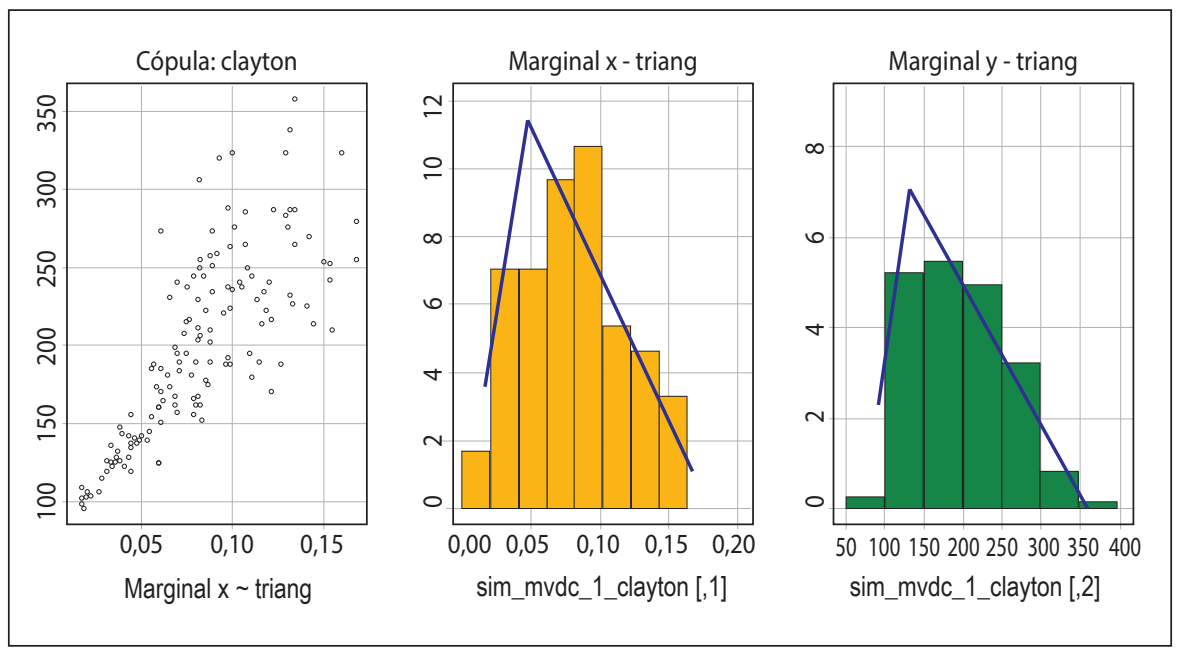

Elaboración propia. 


\section{Gráfico 3}

\section{Gráficos de contorno}
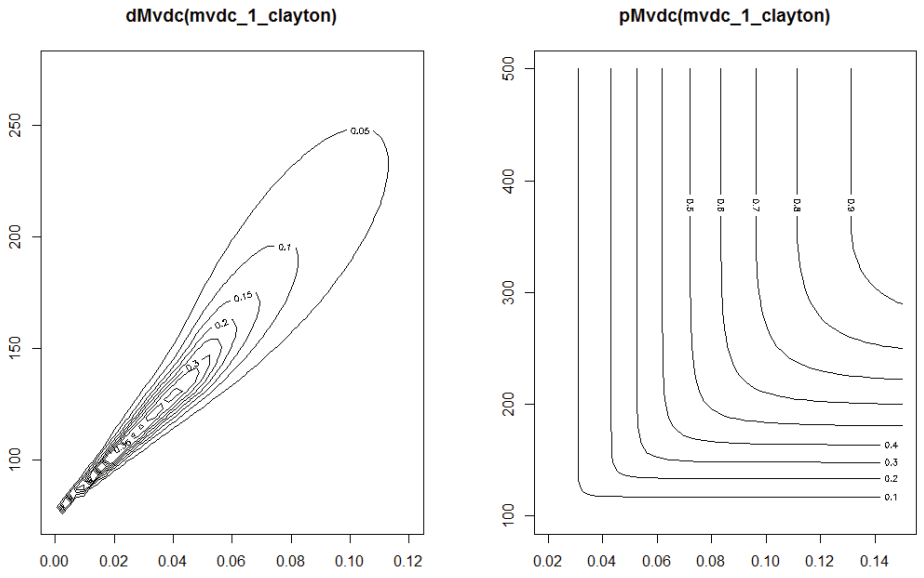

Elaboración propia.

Gráfico 4

Perspectiva de la superficie

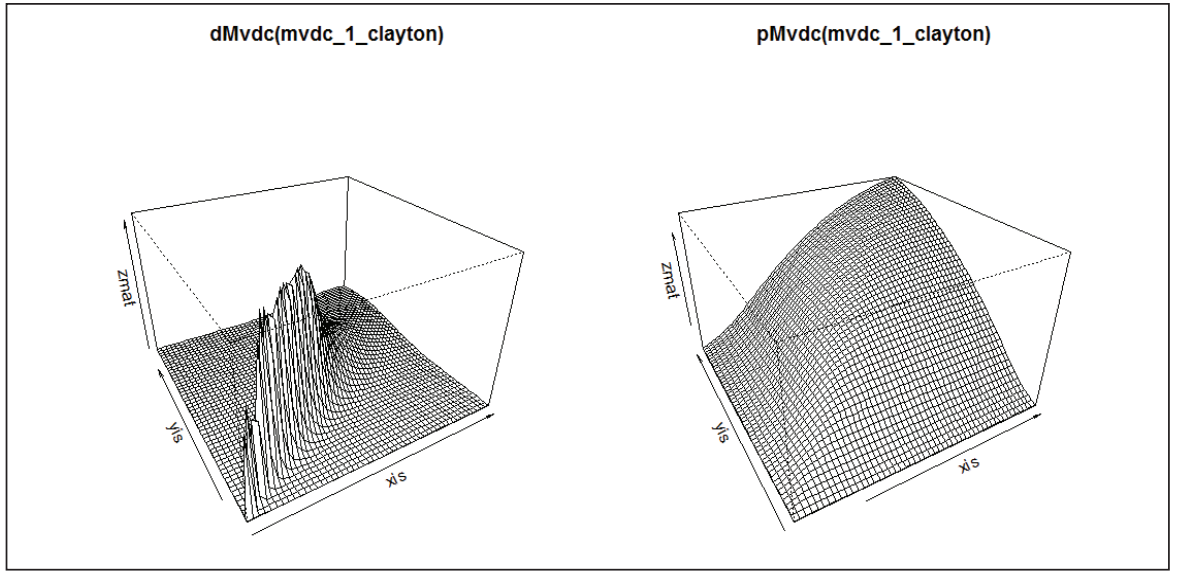

Elaboración propia. 
Para medir el ajuste de los MVDC se realizó la prueba de Genest y Rémillard (2006). Esta prueba utiliza un proceso basado en la cópula empírica para medir el grado de relación entre las distribuciones marginales de las simulaciones generadas por los MVDC. Así $H o:(\mathrm{X}, \mathrm{Y}) \sim(C(u, v)=u v$; es decir, existe independencia entre las distribuciones marginales. La prueba se realiza con el estadístico Global Cramer-von Mises. Los resultados revelan la dependencia de los MVDC y, por tanto, un buen ajuste.

\section{Tabla 4}

\section{Prueba de dependencia multivariada}

\begin{tabular}{|c|c|c|c|}
\hline Grupo & Cópula & Global Cramer-von Mises statistic & p-valor \\
\hline G1 & Clayton & 1,1703 & 0,0005 \\
\hline G2 & Clayton & 1,3783 & 0,0005 \\
\hline G3 & Clayton & 0,0628 & 0,0385 \\
\hline G4 & Clayton & 0,7795 & 0,0005 \\
\hline G5 & Gumbel & 0,1447 & 0,0015 \\
\hline G6 & Clayton & 1,5898 & 0,0005 \\
\hline G7 & Clayton & 1,2415 & 0,0005 \\
\hline G8 & Clayton & 1,0958 & 0,0005 \\
\hline
\end{tabular}

Elaboración propia.

\section{Discusión de resultados}

Para estimar los indicadores de riesgo del portafolio, se sumó el valor de las pérdidas de cada grupo en cada escenario obtenido con los MVDC. Así, se obtuvo una distribución de pérdidas única con lo cual se estimaron los siguientes indicadores: 
Tabla 5

Indicadores de riesgo*

\begin{tabular}{|l|c|c|}
\hline \multirow{2}{*}{\multicolumn{1}{|c|}{ Indicador de riesgo }} & \multicolumn{2}{c|}{ Nivel de confianza } \\
\cline { 2 - 3 } & $\mathbf{9 5 , 0 0} \%$ & $\mathbf{9 9 , 5 0} \%$ \\
\hline Pérdida esperada & \multicolumn{2}{|c|}{2,77} \\
\hline Max. pérdida & 7,10 & 21,42 \\
\hline Credit VaR & 4,33 & 18,65 \\
\hline Expected Shortfall & 7,49 & 21,49 \\
\hline
\end{tabular}

*En millones de dólares.

Elaboración propia.

Los resultados indican una pérdida esperada (PE) de USD 2,77 millones. Con respecto a la PE se observa que los valores del resto de los indicadores son superiores, lo cual representa una alerta para la institución financiera, en especial para el desarrollo de políticas para mitigar el riesgo, pues la PE es el principal indicador sobre el cual los organismos de control ejercen supervisión y es el principal referente para el cálculo de las provisiones de créditos incobrables. En un escenario negativo, la subestimación de la PE conlleva a pérdidas que afectan la rentabilidad de la cartera y comprometen la solidez de la institución debido a la necesidad de liquidez que obliga a la búsqueda de fuentes alternativas de financiamiento o, en el peor de los casos, a la venta de activos financieros.

En particular, al 99,50 \% de confianza la diferencia entre la PE y ES del portafolio es USD 18,71 millones; cifra que representa el monto potencial de pérdidas que no se evidencian en los modelos tradicionales ni se incluyen dentro de la metodología de la Superintendencia de Bancos (SB) y, por tanto, no constan en el análisis de la institución financiera. Al 99,5\% de confianza, el portafolio puede presentar una pérdida máxima de USD 21,42 millones. En condiciones normales de mercado en un mes, la máxima pérdida que puede presentar el portafolio es USD 18,65 millones (Credit VaR). De modificarse algún factor y empeorarse las condiciones de mercado, una vez superada la máxima pérdida, la pérdida esperada luego del Credit VaR puede alcanzar un valor de USD 21,49 millones.

Representando gráficamente el Credit VaR y Expected Shortfall se observa de mejor manera las distancias entre estos indicadores. Adicionalmente, se aprecia la función de densidad de las pérdidas estimadas que concentran 
hasta un monto aproximado de USD 8 millones (96\% del comportamiento del portafolio). El gráfico 5 muestra como, dada la estructura de dependencia en los grupos cuatro, cinco y en especial en el grupo seis, ${ }^{6}$ se producen valores extremos, lo cual a nivel agregado del portafolio se traduce también en valores extremos e indicadores por encima de la PE, evidenciando debilidades y falencias en la administración del riesgo.

Gráfico 5

Densidad pérdidas estimadas, Credit VaR

y Expected Shortfall (miles de USD)

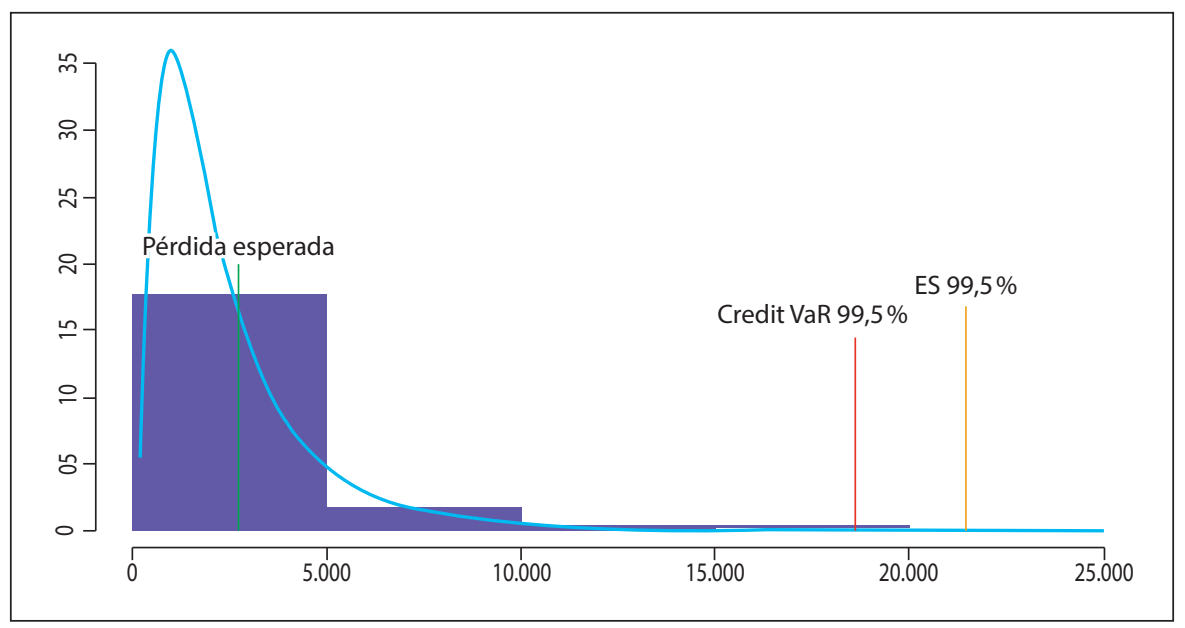

Elaboración propia.

Los resultados, además de alertar en cuanto a la estimación de pérdidas del portafolio, muestran los factores principales donde la institución financiera debería intervenir para mitigar y reducir el riesgo del portafolio. De manera específica, conociendo estos resultados la institución debería prestar atención a las medidas de riesgo de cada grupo, para así desarrollar y enfo-

6. Los valores extremos de los grupos cinco y seis tienen una participación importante sobre los resultados agregados del portafolio, por cuanto poseen los mayores montos concedidos y, por tanto, los mayores montos de exposición. 
car políticas específicas en los procesos de colocación y recuperación de las operaciones de crédito.

Por otra parte, en Ecuador el cálculo de la PE no se realiza considerando el análisis de la estructura ni clase de dependencia que podrían presentar diversos factores de riesgo dentro del portafolio, sino únicamente tomando la fórmula de la normativa de la Superintendencia de Bancos (SB). ${ }^{7}$ A fin de comparar los resultados de los MVDC con los resultados de la SB, se estimaron indicadores con ambas metodologías. La evidencia demuestra que los indicadores obtenidos con la metodología de la SB subestiman los resultados, lo cual implica menores montos de provisión en el portafolio. Esta situación demuestra la capacidad de los MDVC para recoger los resultados extremos, capacidad que no posee la metodología de la SB. Queda así demostrada la pertinencia y necesidad de aplicar la metodología propuesta.

Tabla 6

Indicadores de riesgo empíricos vs. MVDC*

\begin{tabular}{|c|c|c|c|c|c|}
\hline \multirow{3}{*}{ Grupo } & \multirow{3}{*}{$\begin{array}{l}\text { Indicador } \\
\text { de riesgo }\end{array}$} & \multicolumn{2}{|c|}{$\begin{array}{l}\text { Resultados con } \\
\text { metodología SB }\end{array}$} & \multicolumn{2}{|c|}{ Resultados MVDC } \\
\hline & & \multicolumn{2}{|c|}{ Nivel de confianza } & \multicolumn{2}{|c|}{ Nivel de confianza } \\
\hline & & $95,00 \%$ & $99,50 \%$ & $95,00 \%$ & $99,50 \%$ \\
\hline \multirow{4}{*}{ G1 } & Pérdida esperada & \multicolumn{2}{|c|}{$\frac{1}{8,47}$} & \multicolumn{2}{|c|}{8,85} \\
\hline & Max. pérdida & 19,79 & 22,6 & 20,1 & 24,27 \\
\hline & Credit VaR & 11,31 & 14,13 & \begin{tabular}{|l|}
11,25 \\
\end{tabular} & 15,42 \\
\hline & Expected Shorfall & 25,79 & 23,36 & 20,23 & 24,28 \\
\hline \multirow{4}{*}{ G2 } & Pérdida esperada & \multicolumn{2}{|c|}{11,59} & \multicolumn{2}{|c|}{14,58} \\
\hline & Max. pérdida & 177,76 & 184,09 & 303,99 & 370,74 \\
\hline & Credit VaR & 61,83 & 68,15 & 158,3 & 225,06 \\
\hline & Expected Shorfall & 197,4 & 184,13 & 305,77 & 370,75 \\
\hline \multirow{4}{*}{ G3 } & Pérdida esperada & \multicolumn{2}{|r|}{15,73} & \multicolumn{2}{|r|}{16,61} \\
\hline & Max. pérdida & 293,73 & 314,75 & \begin{tabular}{l|l|}
328,2 \\
\end{tabular} & 406,69 \\
\hline & Credit VaR & 136,46 & 157,48 & 162,13 & 240,62 \\
\hline & Expected Shorfall & 338,46 & 314,86 & 329,88 & 406,72 \\
\hline
\end{tabular}

7. Pérdida esperada $=$ nivel de exposición $*$ severidad $*$ probabilidad de incumplimiento. 


\begin{tabular}{|c|c|c|c|c|c|}
\hline \multirow{3}{*}{ Grupo } & \multirow{3}{*}{$\begin{array}{l}\text { Indicador } \\
\text { de riesgo }\end{array}$} & \multicolumn{2}{|c|}{$\begin{array}{l}\text { Resultados con } \\
\text { metodología SB }\end{array}$} & \multicolumn{2}{|c|}{ Resultados MVDC } \\
\hline & & \multicolumn{2}{|c|}{ Nivel de confianza } & \multicolumn{2}{|c|}{ Nivel de confianza } \\
\hline & & $95,00 \%$ & $99,50 \%$ & $95,00 \%$ & $99,50 \%$ \\
\hline \multirow{4}{*}{ G4 } & Pérdida esperada & \multicolumn{2}{|r|}{16,65} & \multicolumn{2}{|r|}{17,33} \\
\hline & Max. pérdida & 409,99 & 460,83 & 589,34 & 1082,6 \\
\hline & Credit VaR & 243,52 & 294,36 & 416,09 & 909,35 \\
\hline & Expected Shorfall & 486,69 & 467,33 & 601,66 & $1.083,26$ \\
\hline \multirow{4}{*}{ G5 } & Pérdida esperada & \multicolumn{2}{|c|}{40,22} & \multicolumn{2}{|c|}{44,12} \\
\hline & Max. pérdida & 673,02 & 684,86 & 920,24 & 1130,26 \\
\hline & Credit VaR & 270,78 & 282,62 & 479,04 & 689,06 \\
\hline & Expected Shorfall & 710,52 & 685,38 & 927,18 & 1130,5 \\
\hline \multirow{4}{*}{ G6 } & Pérdida esperada & \multicolumn{2}{|c|}{27,57} & \multicolumn{2}{|c|}{15,64} \\
\hline & Max. pérdida & 558,04 & 614,02 & 5598,4 & $19.683,3$ \\
\hline & Credit VaR & 282,32 & 338,3 & 4034,06 & $18.118,95$ \\
\hline & Expected Shorfall & 669,31 & 627,58 & 6006,69 & $19.751,75$ \\
\hline \multirow{4}{*}{ G7 } & Pérdida esperada & & 11,39 & \multicolumn{2}{|r|}{14,85} \\
\hline & Max. pérdida & 161,83 & 172,85 & 329,24 & 380,12 \\
\hline & Credit VaR & 47,97 & 59,00 & 180,77 & 231,65 \\
\hline & Expected Shorfall & 183,32 & 173,86 & 330,9 & 380,17 \\
\hline \multirow{4}{*}{ G8 } & Pérdida esperada & \multicolumn{2}{|c|}{96,34} & \multicolumn{2}{|c|}{126,16} \\
\hline & Max. pérdida & 153,03 & 162,77 & 276,22 & 315,46 \\
\hline & Credit VaR & 56,69 & 66,43 & 150,06 & 189,3 \\
\hline & Expected Shorfall & 173,76 & 162,92 & 277,56 & 315,51 \\
\hline
\end{tabular}

*En miles de dólares.

Elaboración propia.

\section{Conclusiones}

En este trabajo se aplicó la teoría de cópulas para conocer la estructura de dependencia del portafolio de crédito; conociendo dicha estructura se pudo estimar indicadores de riesgo que permitieron evidenciar falencias no identificadas con las metodologías tradicionales, permitiendo así direccionar adecuadamente los puntos donde se podría intervenir para reducir y mitigar el riesgo. 
Comparando la PE del portafolio con los resultados de los indicadores de riesgo al 99,50\% de confianza, se apreció una importante debilidad en la administración del riesgo, por cuanto todos los indicadores son superiores a la pérdida esperada estimada.

Al no considerar la estructura de dependencia dentro del portafolio siguiendo la metodología de la SB, se subestima a PE y los eventos de pérdida extrema, lo que afecta directamente en los montos de provisión. Al no realizar una provisión suficiente se podría incurrir en pérdidas, por cuanto esos recursos podrían estar en nuevas colocaciones o inversiones que no estarían disponibles inmediatamente frente a necesidades de liquidez, lo cual conllevaría a la venta de activos financieros por debajo de su valor real.

Los resultados obtenidos son consistentes con los trabajos de Liu (2011), Choe y Jang (2011), Iscoe et al. (2012), Maldonado y Pazmiño (2008) y Luo, Lu y Li (2015) por cuanto, analizando la estructura de dependencia del portafolio con la cópula de Arquímedes puntualmente con la de Clayton, se cuantifican mejor los indicadores de riesgo, en especial los de riesgo extremo.

Este documento se desarrolló con cópulas bivariadas; a futuro se podría fortalecer el análisis con cópulas multivariadas. También se podría incluir en el estudio el criterio de probabilidad de ocurrencia de los indicadores de riesgo.

\section{Referencias}

Bickel, Peter, Peter Diggle, Stephen Fienberg, Ursula Gather, Ingram Olkin y Scott Zeger. 1991. An Introduction to Copulas. Portland: Springer Series in Statistics.

Boyé, Erick, Ashkan Niikeghbali, Valdo Durrleman, Gaël Riboulet y Thierry Roncalli. 2000. "Copulas for Finance. A Reading Guide and Some Applications". International Conference in Finance. Londres. Accedido 18 de abril. http://ssrn.com/paper=1032533.

Braxton, Peter, y Travis Manning. 2011. "Probability Distributions for Risk Analysis". Washington D. C. Cost Estimating and Analysis Association. Accedido 18 de abril. https://bit.ly/2PFVt6a.

Chang, Jow-ran, y An Chi Chen. 2010. "Copula, Correlated Defaults and Credit VaR". En Handbook of Quantitative Finance and Risk Management, editado por Cheng Few Lee y John Lee, 697-711. Boston: Springer. https://doi.org/https://doi.org/10.1007/978-0-38777117-5_46. 
Chen, James Ming. 2018. “On Exactitude in Financial Regulation: Value-at-Risk, Expected Shortfall, and Expectiles”. Risks 6 (2): 3-20. https://doi.org/10.3390/risks6020061.

Cherubini, Umberto, Elisa Luciano y Walter Vecchiato. 2004. Copula Methods in Finance. Nueva Jersey: Wiley Finance Series.

Choe, Geon H., y Jang Hyun Jin. 2011. "Efficient Algorithms for Basket Default Swap Pricing with Multivariate Archimedean Copulas". Insurance: Mathematics and Economics 48 (2): 205-213. https://bit.ly/3enZeHT.

Choudhry, Moorad. 2006. An Introduction Value-At-Risk. West Sussex: John Wiley \& Sons Ltd.

Clemente, Annalisa Di, y Claudio Romano. 2004. "Measuring and Optimizing Portfolio Credit Risk: A Copula-Based Approach”. Economic Notes 33 (3): 325-357. https://doi.org/10 .1111/j.0391-5026.2004.00135.

Díaz Hernández, Adán, y José Carlos Ramírez. 2009. “Una metodologia basada en cópulas y valores extremos para estimar el capital económico requerido de un portafolio de créditos al menudeo". Revista de Análisis Económico 24 (2): 95-132. http://dx.doi.org/10.4067/ S0718-88702009000200004.

EC. 2004. Libro I.- Normas Generales para la aplicación de la Ley General de Instituciones del Sistema Financiero.- Título X.- De la gestión y administración de riesgos. Capítulo II de la Administración del Riesgo de Crédito. Registro Oficial 250, Suplemento, 13 de enero.

Edinson, Caicedo, Claramunt M. Mercè y Monserrat Casanovas. 2011. "Medición del riesgo de crédito mediante modelos estructurales: una aplicación al mercado colombiano". Cuadernos de Administación 24 (42): 73-100. https://bit.ly/3c88TlE.

Embrechts, Paul, Alexander Mcneil y Daniel Straumann. 1999. "Correlation: Pitfalls and Alternatives”. Risk Magazine 12 (5): 69-71. https://bit.ly/2FOWtTD.

Fiorito, Fabián. 2006. "La simulación como una herramienta para el manejo de la incertidumbre”. Tesis de posgrado, Universidad del CEMA, Buenos Aires. https://bit.ly/3285mk7.

Frey, Rüdiger, y Alexander McNeil. 2002. "VaR and Expected Shortfall in Portfolios of Dependent Credit Risks: Conceptual and Practical Insights". Journal of Banking and Finance 26 (7): 1317-1334. https://doi.org/https://doi.org/10.1016/S0378-4266(02)00265-0.

Genest, Christian, Bruno Remillard y David Beaudoin. 2009. "Goodness-of-Fit Tests for Copulas: A Review and a Power Study”. Mathematics and Economics 44 (2): 199-213. https://doi.org/10.1016/j.insmatheco.2007.10.005.

Hull, John. 2007. "VAR versus Expected Shortfall”. Risk Management. Accedido 25 de mayo 2020. https://bit.ly/VAR_ES_HULL.

Iscoe, Ian, Alexander Kreinin, Helmut Mausser y Oleksandr Romanko. 2012. "Portfolio Credit-risk Optimization". Journal of Banking \& Finance 36 (6): 1604-1615. https://bit.ly/ Iscoe_Credit_risk.

Jadhav, Deepak, y T. V. Ramanathan. 2009. "Parametric and Non-Parametric Estimation of Value-at-Risk". The Journal of Risk Model Validation 3 (1): 51-71. 10.21314/ JRMV.2009.034. 
Li, David X. 1999. “On Default Correlation: A Copula Function Approach”. The Journal of Fixed Income 9 (4): 43-54. https://doi.org/http://dx.doi.org/10.2139/ssrn.187289.

Li, Ping, Xiaoxu Wang y Haibo Wang. 2013. "A Factor Model for the Calculation of Portfolio Credit VaR". Procedia Computer Science 17: 611-618. https://doi.org/10.1016/j. procs.2013.05.079.

Liu, Jiubiao. 2011. "Coherent Measures of Credit Portfolio Risk Based on T-Copula”. Journal of Beijing University of Aeronautics and Astronautics 24 (1): 81-85. https://bit.ly/2FvZUiF.

Luo, Changqing, Yanlin Lu y Mengzhen Li. 2015. "Credit Portfolio Risk Evaluation Based on the Pair Copula VaR Models". Journal of Finance and Economics 3 (1): 15-30. https:// doi.org/10.12735/jfe.v3i1p15.

Maldonado, Diego, y Mariela Pazmiño. 2008. "Nuevas herramientas para la administración del riesgo crediticio: el caso de una cartera crediticia ecuatoriana". Cuestiones Económicas 24 (2): 5-75. https://bit.ly/35XOijh.

McNeil, Alexander J., Frey Rudiger y Mark A. Nyfeler. 2001. "Copulas and Credit Models". Risk 10: 111-114. https://bit.ly/32C2Y1P.

McNeil, Alexander J.; Frey Rüdiger y Paul Embrechts. 2005. Quantitative Risk Management. Nueva Jersey: Princeton University Press.

Melo, Luis, y Oscar Becerra. 2008. "Medidas de riesgo financiero usando cópulas: teoría y aplicaciones". Borradores de Economía 489: 1-93. 10.32468/be.489.

Mora, Andrés. 2010. "Una propuesta de creditmetrics y expected shortfall para medición de riesgo de crédito". Revista Civilizar de Empresa y Economía 1 (2): 104-125. https://doi. org/10.22518/2462909X.64.

Osei, Price, y Adam Anoke. 2020. "Copula-Based Assessment of Co-Movement and Tail Dependence Structure Among Major Trading Foreign Currencies in Ghana". Risks 8 (2): 4-20. 10.3390/risks8020055.

Plascencia, Tania. 2012. "Valor en riesgo utilizando cópulas financieras: aplicación al tipo de cambio mexicano (2002-2011)". Contabilidad y Negocios 7 (14): 57-68. https://bit.ly/337u4Ak.

Romano, Claudio. 2002. Applying Copula Funtion to Risk Management. Bucarest: Banca Roma.

Sahamkhadam, Maziar, Andreas Stephan y Ralf Östermark. 2018. "Portfolio Optimization Based on GARCH-EVT-Copula Forecasting Models". International Journal of Forecasting 34 (3): 497-506. https://doi.org/10.1016/j.ijforecast.2018.02.004.

Sheppard, Kevin. 2009. "Value-at-Risk, Expected Shortfall and Density Forecasting”. Accedido 27 de abril. https://bit.ly/2WYAcJe.

Szetela, Beata, Grzegorz Mentel y Jacek Brożyna. 2019. "Modelling European Sovereign Default Probabilities with Copulas". Economic Research-Ekonomska Istrazivanja 32 (1): 1716-1726. https://doi.org/10.1080/1331677X.2019.1629325.

Trabelsi, Nader, y Aviral Kumar Tiwari. 2019. "Market-Risk Optimization among the Developed and Emerging Markets with CVaR Measure and Copula Simulation”. Risks 7 (22): 1-20. https://doi.org/10.3390/risks7030078. 